\title{
Front Matter: Volume 6518
}

, "Front Matter: Volume 6518," Proc. SPIE 6518, Metrology, Inspection, and Process Control for Microlithography XXI, 651801 (21 April 2009); doi: $10.1117 / 12.736738$

SPIE. Event: SPIE Advanced Lithography, 2007, San Jose, California, United States 


\section{PROCEEDINGS OF SPIE}

\section{Metrology, Inspection, and Process Control for Microlithography XXI}

Chas N. Archie

Editor

26 February-1 March 2007

San Jose, California, USA

Sponsored and Published by

SPIE-The International Society for Optical Engineering

Cooperating Organization

SEMATECH (USA)

Volume 6518

Part One of Three Parts

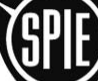

The International Society for Optical Engineering

Proceedings of SPIE-The International Society for Optical Engineering, 9780819466372, v. 6518

SPIE is an international technical society dedicated to advancing engineering and scientific applications of optical, photonic, imaging, electronic, and optoelectronic technologies. 
The papers included in this volume were part of the technical conference cited on the cover and title page. Papers were selected and subject to review by the editors and conference program committee. Some conference presentations may not be available for publication. The papers published in these proceedings reflect the work and thoughts of the authors and are published herein as submitted. The publisher is not responsible for the validity of the information or for any outcomes resulting from reliance thereon.

Please use the following format to cite material from this book:

Author(s), "Title of Paper," in Metrology, Inspection, and Process Control for Microlithography XXI, edited by Chas N. Archie, Proceedings of SPIE Vol. 6518 (SPIE, Bellingham, WA, 2007) Article CID Number.

ISSN 0277-786X

ISBN 9780819466372

Published by

SPIE-The International Society for Optical Engineering

P.O. Box 10, Bellingham, Washington 98227-0010 USA

Telephone 1 360/676-3290 (Pacific Time) · Fax 1 360/647-1445

http://www.spie.org

Copyright (C) 2007, The Society of Photo-Optical Instrumentation Engineers

Copying of material in this book for internal or personal use, or for the internal or personal use of specific clients, beyond the fair use provisions granted by the U.S. Copyright Law is authorized by SPIE subject to payment of copying fees. The Transactional Reporting Service base fee for this volume is $\$ 18.00$ per article (or portion thereof), which should be paid directly to the Copyright Clearance Center (CCC), 222 Rosewood Drive, Danvers, MA 01923. Payment may also be made electronically through CCC Online at http://www.copyright.com. Other copying for republication, resale, advertising or promotion, or any form of systematic or multiple reproduction of any material in this book is prohibited except with permission in writing from the publisher. The CCC fee code is 0277$786 \times / 07 / \$ 18.00$.

Printed in the United States of America. 


\title{
Contents
}

\section{Part One}

\author{
xix Symposium Committee \\ xxi Conference Committee \\ xxiii Plenary Paper: Lithography beyond $32 \mathrm{~nm}$ : a role for imprint? M. Melliar-Smith, Molecular \\ Imprints (USA)
}

\section{SESSION 1 KEYNOTE SESSION}

651802 Metrology challenges of double exposure and double patterning (Keynote Paper) [6518-01]

W. H. Arnold, M. Dusa, ASML Technology Development Ctr. (USA); J. Flinders, ASML Holding, B. V. (Netherlands)

\section{SESSION 2 PROCESS CONTROL I: LITHOGRAPHY CONTROL}

651803 Process monitor gratings (Invited Paper) [6518-02]

T. A. Brunner, C. P. Ausschnitt, IBM, SRDC (USA)

651804 Lithography process control using scatterometry metrology and semi-physical modeling [6518-03]

K. Lensing, J. Cain, A. Prabhu, A. Vaid, R. Chong, R. Good, B. LaFontaine, O. Kritsun, Advanced Micro Devices (USA)

651805 Comparison of back side chrome focus monitor to focus self-metrology of an immersion scanner [6518-131]

K. D'havé, T. Machida, D. Laidler, S. Cheng, IMEC (Belgium)

651806 Evaluating a scatterometry-based focus monitor technique for hyper-NA lithography [6518-05]

C. S. Saravanan, S. Nirmalgandhi, Nanometrics, Inc. (USA); O. Kritsun, A. Acheta,

R. Sandberg, B. La Fontaine, H. J. Levinson, K. Lensing, AMD (USA); M. Dusa, J. Hauschild,

A. Pici, ASML US, Inc. (USA)

\footnotetext{
Pagination: Proceedings of SPIE follow an e-First publication model, with papers published first online and then in print and on CD-ROM. Papers are published as they are submitted and meet publication criteria. A unique, consistent, permanent citation identifier (CID) number is assigned to each article at the time of the first publication. Utilization of CIDs allows articles to be fully citable as soon they are published online, and connects the same identifier to all online, print, and electronic versions of the publication.

SPIE uses a six-digit CID article numbering system in which:

- The first four digits correspond to the SPIE volume number.

- The last two digits indicate publication order within the volume using a Base 36 numbering system employing both numerals and letters. These two-number sets start with 00, 01, 02, 03, 04, 05, 06, 07, 08, 09, OA, OB ... 0Z, followed by 10-1Z, 20-2Z, etc.

The CID number appears on each page of the manuscript. The complete citation is used on the first page, and an abbreviated version on subsequent pages.
} 
651807 Focus and dose controls, and their application in lithography [6518-06]

H. Ina, K. Sentoku, S. Oishi, T. Miyashita, T. Matsumoto, Canon, Inc. (Japan)

SESSION 3 CD FOR DEVELOPMENT I: OPC AND METROLOGY

651808 Statistical optimization of sampling plan and its relation to OPC model accuracy [6518-07]

G. Han, A. Brendler, S. Mansfield, J. Meiring, IBM Microelectronics (USA)

651809 Automatic setup of in-line critical dimension (CD) recipes during OPC qualification in a foundry environment [6518-08]

Y. Yudhistira, Q. S. Fong, C. S. Sun, K. H. Peng, Chartered Semiconductor Manufacturing, Ltd. (Singapore); R. Ren, S. L. Ng, Applied Materials South East Asia Pte., Ltd. (Singapore); A. Siany, S. Levi, Applied Materials Israel (Israel)

65180A Quantification of two-dimensional structures generalized for OPC model verification [6518-09]

X. Shi, J. F. Chen, D. Van Den Broeke, S. Hsu, M. Hsu, ASML MaskTools (USA)

65180B Setting MRC rules: balancing inspection capabilities, defect sensitivity, and OPC [6518-10] I. Stobert, J. Bruce, IBM Semiconductor Research and Development Ctr. (USA); M. Gheith, A. Seoud, Mentor Graphics Corp. (USA)

65180C Methodology to set up accurate OPC model using optical CD metrology and atomic force microscopy [6518-11]

Y.-A. Shim, J. Kang, S.-U. Lee, J. Kim, K. Kim, DongbuElectronics (South Korea)

65180D SEM-contour-based OPC model calibration through the process window [6518-12] J. Vasek, Freescale Semiconductor, Inc. (USA); O. Menedeva, D. Levitzky, O. Lindman, Y. Nemadi, Applied Materials, Inc. (Israel); G. E. Bailey, J. L. Sturtevant, Mentor Graphics Corp. (USA)

\section{SESSION 4 OVERLAY}

65180 Meeting overlay requirements for future technology nodes with in-die overlay metrology (Invited Paper) [6518-13]

B. Schulz, AMD Saxony LLC and Co. KG (Germany); R. Seltmann, AMD Fab36 LLC and Co. KG (Germany); J. Busch, AMD Saxony LLC and Co. KG (Germany); F. Hempel, AMD Fab36 LLC and Co. KG (Germany); E. Cotte, AMTC GmbH and Co. KG (Germany); B. Alles, Univ. of Technology Munich (Germany)

65180F Zero-order imaging of device-sized overlay targets using scatterfield microscopy [6518-14] B. M. Barnes, National Institute of Standards and Technology (USA); L. P. Howard, National Institute of Standards and Technology (USA) and Precera, Inc. (USA); J. Jun, National Institute of Standards and Technology (USA); P. Lipscomb, ISMI SEMATECH (USA);

R. M. Silver, National Institute of Standards and Technology (USA)

65180G Blossom overlay metrology implementation [6518-15]

C. P. Ausschnitt, W. Chu, D. Kolor, J. Morillo, J. L. Morningstar, W. Muth, C. Thomison, R. J. Yerdon, IBM (USA); L. A. Binns, P. Dasari, H. Fink, N. P. Smith, G. Ananew, Nanometrics, Inc. (USA) 
$65180 \mathrm{H}$ The application of SMASH alignment system for 65-55-nm logic devices [6518-16] M. Miyasaka, H. Saito, T. Tamura, T. Uchiyama, NEC Electronics Corp. (Japan); P. Hinnen, H.-W. Lee, M. van Kemenade, M. Shahrjerdy, R. van Leeuwen, ASML (Netherlands)

651801 Overlay metrology tool calibration [6518-17]

L. A. Binns, P. Dasari, N. P. Smith, G. Ananew, H. Fink, Nanometrics, Inc. (USA);

C. P. Ausschnitt, J. Morningstar, C. Thomison, R. J. Yerdon, IBM SRDC (USA)

65180J Improved overlay control through automated high order compensation [6518-105] S. Wakamoto, Y. Ishii, K. Yasukawa, A. Sukegawa, Nikon Corp. (Japan); S. Maejima, Renesas Technology Corp. (Japan); A. Kato, KLA-Tencor Japan, Ltd. (Japan);

J. C. Robinson, B. J. Eichelberger, KLA-Tencor Corp. (USA); P. Izikson, M. Adel, KLA-Tencor Corp. (Israel)

SESSION 5 CDSEM: TECHNIQUES, LIMITS, ETC.

65180K Monte Carlo modeling of secondary electron imaging in three dimensions (Invited Paper) [6518-19]

J. S. Villarrubia, N. W. M. Ritchie, J. R. Lowney, National Institute of Standards and Technology (USA)

65180L Evaluation of CD-SEM measurement uncertainty using secondary electron simulation with charging effect [6518-20]

H. Abe, A. Hamaguchi, Y. Yamazaki, Toshiba Corp. (Japan)

65180N Carbon nanotube metrology in a CD SEM [6518-22]

C. Yates, ON Semiconductor, Inc. (USA); T. Rueckes, Nantero, Inc. (USA); R. J. Carter, LSI Logic Corp. (USA)

651800 Physical matching versus CD matching for CD SEM [6518-23]

R. Kris, G. Zuckerman, E. Sommer, Z. Hadad, S. Dror, A. Tam, N. Shcolnik, Applied Materials Israel (Israel)

\section{SESSION 6 INSPECTION}

65180P Developing the new ADC algorithm that enables to identify the defect source [6518-24] P.-Y. Tsai, W.-F. Chiu, T.-Y. Chen, Taiwan Semiconductor Manufacturing Co., Ltd. (Taiwan); F. Endo, Y. Kariya, K. Nemoto, Hitachi High-Technologies Corp. (Japan)

65180Q Developing micro ADI methodology for new litho process monitoring strategies [6518-25] I. Mäge, U. Seifert, Qimonda AG (Germany); B. Saville, M. Tuckermann, KLA-Tencor GmbH (Germany)

65180S Immersion lithography defectivity analysis at DUV inspection wavelength [6518-27]

E. Golan, D. Meshulach, N. Raccah, J. H. Yeo, O. Dassa, Applied Materials (Israel); S. Brandl, C. Schwarz, Qimonda, North America Corp. (USA); B. Pierson, ASML (USA); W. Montgomery, Albany NanoTech Complex (USA)

65180T Innovative metrology for wafer edge defectivity in immersion lithography [6518-28] I. Pollentier, IMEC (Belgium); F. Iwamoto, Matsushita Electric Industrial Co., Ltd. (Japan); M. Kocsis, Intel Corp. (USA); A. Somanchi, F. Burkeen, S. Vedula, KLA-Tencor Corp. (USA) 
$65180 \mathrm{U}$ Fundamental limits of optical critical dimension metrology: a simulation study (Invited Paper) [6518-29]

R. Silver, T. Germer, R. Attota, B. M. Barnes, National Institute of Standards and Technology (USA); B. Bunday, J. Allgair, International Sematech Manufacturing Initiative (USA); E. Marx, J. Jun, National Institute of Standards and Technology (USA)

$65180 \mathrm{~V}$ Detailed analysis of capability and limitations of CD scatterometry measurements for 65- and 45-nm nodes [6518-30]

I. Pundaleva, R. Chalykh, J. Lee, S. Choi, W. Han, Samsung Electronics Co., Ltd. (South Korea)

65180W Real-time profile shape reconstruction using dynamic scatterometry [6518-31] S. Soulan, M. Besacier, LTM-CNRS (France); T. Leveder, CEA-LETI (France); P. Schiavone, LTM-CNRS (France)

65180X Mueller polarimetry in the back focal plane [6518-32]

A. De Martino, S. Ben Hatit, M. Foldyna, LPICM-CNRS, Ecole Polytechnique (France)

$65180 Z$ Modeling the effect of line profile variation on optical critical dimension metrology [6518-34]

T. A. Germer, National Institute of Standards and Technology (USA)

\section{SESSION 8 STANDARDS AND TECHNIQUES}

651810 TEM calibration methods for critical dimension standards [6518-35]

N. G. Orji, R. G. Dixson, National Institute of Standards and Technology (USA):

D. I. Garcia-Gutierrez, Advanced Technology Development Facility (USA); B. D. Bunday, M. Bishop, International SEMATECH Manufacturing Initiative (USA); M. W. Cresswell, R. A. Allen, National Institute of Standards and Technology (USA); J. A. Allgair, International SEMATECH Manufacturing Initiative (USA)

651811 Image simulation and surface reconstruction of undercut features in atomic force microscopy [6518-36]

X. Qian, Illinois Institute of Technology (USA); J. Villarrubia, National Institute of Standards and Technology (USA); F. Tian, Illinois Institute of Technology (USA); R. Dixson, National Institute of Standards and Technology (USA)

651812 Statistical approach utilizing neural networks for CD error prediction [6518-37] M. Asano, M. Satake, S. Tanaka, S. Mimotogi, Toshiba Corp. (Japan)

651813 Characterizing pattern structures using x-ray reflectivity [6518-38]

H.-J. Lee, C. L. Soles, H. W. Ro, S. Kang, E. K. Lin, A. Karim, W. Wu, National Institute of Standards and Technology (USA); D. R. Hines, Univ. of Maryland (USA)

651814 Accuracy in optical image modeling [6518-39]

J. Potzick, E. Marx, National Institute of Standards and Technology (USA); M. Davidson, Spectel Research Corp. (USA) 
651815 Single crystal critical dimension reference materials (SCCDRM): process optimization for the next generation of standards [6518-40]

R. G. Dixson, W. F. Guthrie, M. Cresswell, R. A. Allen, N. G. Orji, National Institute of

Standards and Technology (USA)

\section{SESSION 9 REFERENCE METROLOGY AND INSTRUMENTS}

651816 Comparison and uncertainties of standards for critical dimension atomic force microscope tip width calibration [6518-41]

R. Dixson, N. G. Orji, National Institute of Standards and Technology (USA)

651818 TEM validation of CD AFM image reconstruction [6518-135]

G. A. Dahlen, L. Mininni, M. Osborn, H.-C. Liu, J. R. Osborne, Veeco Instruments, Inc. (USA);

B. Tracy, A. del Rosario, Spansion, Inc. (USA)

651819 Application of carbon nanotube probes in a critical dimension atomic force microscope [6518-43]

B. C. Park, Korea Research Institute of Standards and Science (South Korea); J. Choi, Korea Research Institute of Standards and Science (South Korea) and Chungnam National Univ. (South Korea); S. J. Ahn, D.-H. Kim, Korea Research Institute of Standards and Science (South Korea); J. Lyou, Chungnam National Univ. (South Korea); R. Dixson, N. G. Orji, J. Fu, T. V. Vorburger, National Institute of Standards and Technology (USA)

$65181 \mathrm{~B}$ In-line AFM characterization of STI profile at the $65 \mathrm{~nm}$ node with advanced carbon probes [6518-45]

M. D. Sardo, STMicroelectronics (France); A. Berthoud, NXP Semiconductors (France);

J.-C. Royer, CEA-LETI Minatec (France); C. Kusch, Nanotools GmbH (Germany)

\section{SESSION 10 METROLOGY CHALLENGES}

65181C Impact of thin film metrology on the lithographic performance of 193-nm bottom antireflective coatings [6518-46]

C. A. Mack, Lithoguru.com (USA); D. Harrison, C. Rivas, P. Walsh, MetroSol, Inc. (USA)

65181D Dielectric-thickness dependence of damage induced by electron-beam irradiation of MNOS gate pattern [6518-47]

M. Matsui, T. Mine, K. Hozawa, K. Watanabe, Hitachi, Ltd. (Japan); J. Inove, H. Nagaishi, Renesas Technology Corp. (Japan)

65181E OPC model data collection for $45-\mathrm{nm}$ technology node using automatic CD-SEM offline recipe creation [6518-48]

D. Fischer, M. Talbi, A. Wei, IBM Semiconductor Research and Development Ctr. (USA);

O. Menadeva, Applied Materials Israel (Israel); R. Cornell, Applied Materials (USA)

$65181 \mathrm{~F}$ Line width measurement below $60 \mathrm{~nm}$ using an optical interferometer and artificial neural network [6518-49]

C. W. See, R. J. Smith, M. G. Somekh, Univ. of Nottingham (United Kingdom); A. Yacoot, National Physical Lab. (United Kingdom) 
$65181 \mathrm{G}$ Metrology challenges for advanced lithography techniques [6518-50]

I. Englard, Applied Materials Europe (Netherlands); P. Vanoppen, J. Finders,

I. Minnaert-Janssen, F. Duray, J. Meessen, G.-J. Janssen, ASML Netherlands (Netherlands);

O. Adan, L. Gershtein, R. Peltinov, C. Masia, Applied Materials Europe (Israel); R. Piech,

Applied Materials Europe (Netherlands)

\section{SESSION $11 \quad$ NEW TRENDS IN METROLOGY}

65181H Transition from precise to accurate critical dimension metrology (Invited Paper) [6518-51]

V. A. Ukraintsev, M. C. Tsai, T. Lii, R. A. Jackson, Texas Instruments, Inc. (USA)

651811 Device metrology with high-performance scanning ion beams [6518-52]

D. C. Joy, Univ. of Tennessee (USA); B. J. Griffin, Univ. of Tennessee (USA) and Univ. of Western Australia (Australia); J. Notte, L. Stern, S. McVey, B. Ward, C. Fenner, ALIS Corp. (USA)

$65181 \mathrm{~J}$ Beyond measurement uncertainty: improving the productivity of metrology tools through recipe error analysis [6518-53]

E. Solecky, A. Bennasser, E. Weissmann, IBM Systems and Technology Group (USA)

65181K Realizing value-added metrology (Invited Paper) [6518-54]

B. Bunday, P. Lipscomb, J. Allgair, D. Patel, International SEMATECH Manufacturing Initiative (USA); M. Caldwell, Freescale (USA); E. Solecky, C. Archie, J. Morningstar, IBM

Microelectronics (USA); B. J. Rice, SEMATECH (USA); B. Singh, J. Cain, I. Emami, Advanced

Micro Devices (USA); B. Banke, IBM Microelectronics (USA); A. Herrera, Spansion (USA);

V. Ukraintsev, J. Schlessinger, J. Ritchison, Texas Instruments (USA)

65181L Improved dimension and shape metrology with versatile atomic force microscopy [6518-138]

M. Caldwell, Freescale Semiconductor, Inc. (USA); T. Bao, Veeco Instruments, Inc. (USA);

J. Hackenberg, B. McLain, O. Munoz, T. Stephens, V. Vartanian, Freescale Semiconductor, Inc. (USA)

65181M Enabling immersion lithography and double patterning [6518-56]

K. M. Monahan, Quantitative Yield Strategies (USA); A. Widmann, KLA-Tencor Corp. (USA)

\section{Part Two}

SESSION 12 LINE-EDGE AND LINE-WIDTH ROUGHNESS

$65181 \mathrm{~N}$ Correlation length and the problem of line width roughness [6518-57]

V. Constantoudis, G. P. Patsis, E. Gogolides, Institute of Microelectronics, NCSR (Greece)

651810 Line edge roughness characterization of sub-50nm structures using CD-SAXS: round-robin benchmark results [6518-195]

C. Wang, R. L. Jones, E. K. Lin, W. Wu, J. S. Villarrubia, K.-W. Choi, National Institute of Standards and Technology (USA); J. S. Clarke, B. J. Rice, M. Leeson, J. Roberts, R. Bristol, Intel Corp. (USA); B. Bunday, International SEMATECH Manufacturing Initiative (USA) 
65181P Characterization of line-edge roughness in Cu/low-k interconnect pattern [6518-59] A. Yamaguchi, D. Ryuzaki, J. Yamamoto, Hitachi, Ltd. (Japan); H. Kawada, T. lizumi, Hitachi High-Technologies Corp. (Japan)

$65181 Q$ Impact of acid diffusion length on resist LER and LWR measured by CD-AFM and CD-SEM [6518-60] J. Foucher, CEA/LETI/MINATEC (France); A. Pikon, C. Andes, J. Thackeray, Rohm and Haas Electronic Materials (USA)

65181R Advanced edge roughness measurement application for mask metrology [6518-61] T. Marschner, J. Richter, U. Dersch, Advanced Mask Technology Ctr. (Germany); A. Moran, R. Katz, D. Chase, R. Falah, T. Coleman, Applied Materials Israel (Israel)

65181S The coming of age of tilt CD-SEM [6518-114]

B. Bunday, J. Allgair, International SEMATECH Manufacturing Initiative (USA); E. Solecky, C. Archie, IBM Microelectronics (USA); N. G. Orji, National Institute of Standards and Technology (USA); J. Beach, Advanced Technology Development Facility (USA); O. Adan, R. Peltinov, M. Bar-zvi, Applied Materials (Israel); J. Swyers, Applied Materials (USA)

\section{SESSION 13 MASK METROLOGY}

$65181 \mathrm{~T}$ Scatterometry on pelliclized masks: an option for wafer fabs [6518-63] E. Gallagher, C. Benson, IBM Corp. (USA); M. Higuchi, Y. Okumoto, Toppan Electronics Inc. (USA); M. Kwon, S. Yedur, S. Li, Timbre Technologies, Inc. (USA); S. Lee, M. Tabet, Nanometrics, Inc. (USA)

$65181 \mathrm{U}$ Development of advanced mask inspection optics with transmitted and reflected light image acquisition [6518-64]

R. Hirano, R. Ogawa, H. Suzuki, K. Takahara, Y. Tsuji, S. Murakami, N. Kikuiri, K. Usuda, Advanced Mask Inspection Technology, Inc. (Japan)

$65181 \mathrm{~V}$ Real time monitoring of reticle etch process tool to investigate and predict critical dimension performance [6518-65]

R. Deming, K. Yung, M. Guglielmana, D. Bald, K. Baik, F. Abboud, Intel Corp. (USA)

$65181 \mathrm{~W}$ CAD-based line/space mix-up prevention for reticle metrology [6518-66]

T. Marschner, M. Enger, F. Ludewig, Advanced Mask Technology Ctr. (Germany); R. Falah, S. Latinsky, O. Lindman, T. Coleman, Applied Materials (Israel)

$65181 \mathrm{X}$ Aspects and new developments on edge angle and edge profile metrology at PTB [6518-67]

B. Bodermann, E. Buhr, A. Diener, Physikalisch-Technische Bundesanstalt (Germany); K. Dirscherl, Danish Fundamental Metrology Ltd. (Denmark); G. Ehret, C. G. Frase, M. Wurm, Physikalisch-Technische Bundesanstalt (Germany)

$65181 Y$ Study of rigorous effects and polarization on phase shifting masks through simulations and in-die phase measurements [6518-68]

K. M. Lee, M. Tavassoli, M. Lau, K. Baik, B. Lieberman, Intel Mask Operation (USA); S. Perlitz,

U. Buttgereit, T. Scherübl, Carl Zeiss SMS GmbH (Germany) 
$65181 \mathrm{Z}$ Advances in process overlay: alignment solutions for future technology nodes [6518-69] H. Megens, R. van Haren, S. Musa, M. Doytcheva, S. Lalbahadoersing, M. van Kemenade, H.-W. Lee, P. Hinnen, F. van Bilsen, ASML (Netherlands)

651820 Algorithm for lithography advanced process control system for high-mix low-volume products [6518-70]

E. Kawamura, Fujitsu. Ltd. (Japan)

$651821 \quad$ Advanced process control with design-based metrology [6518-71]

H. Yang, J. Kim, J. Hong, D. Yim, J. Kim, Hynix Semiconductor, Inc. (South Korea); T. Hasebe, M. Yamamoto, NanoGeometry Research, Inc. (Japan)

651822 Investigation of optimized wafer sampling with multiple integrated metrology modules within photolithography equipment [6518-72]

T. L. Taylor, Micron Technology, Inc. (USA); E. Makimura, Tokyo Electron America, Inc. (USA)

651823 Advanced lithography parameters extraction by using scatterometry system [6518-73] W. Zhou, M. Tang, H. Koh, M. Zhou, Chartered Semiconductor Manufacturing, Ltd.

(Singapore)

\section{SESSION 15 CD FOR DEVELOPMENT II}

651824 Line edge roughness impact on critical dimension variation [6518-74]

Y. Ma, H. J. Levinson, T. Wallow, Advanced Micro Devices, Inc. (USA)

651825 Predicting electrical measurements by applying scatterometry to complex spacer structures [6518-75]

M. Sendelbach, J. Ayala, IBM Microelectronics (USA); P. Herrera, KLA-Tencor Corp. (USA)

651826 Characterization of bending CD errors induced by resist trimming in $65 \mathrm{~nm}$ node and beyond [6518-76]

Y. GU, J. B. Friedmann, V. Ukraintsev, G. Zhang, T. Wolf, T. Lii, R. Jackson, Texas Instruments, Inc. (USA)

651827 Characterization of capacitive 3D deep trench mask open structures using scatterometry [6518-78]

S. Zangooie, IBM Microelectronics (USA); P. Herrera, KLA-Tencor (USA); A. Mesfin, C. Archie, M. Sendelbach, IBM Microelectronics (USA)

\section{POSTER SESSION}

651829 Application of perturbation methods in optical scatterometry [6518-33]

B. C. Bergner, T. J. Suleski, Univ. of North Carolina, Charlotte (USA)

65182B SEM metrology for advanced lithographies [6518-62]

B. Bunday, J. Allgair, International SEMATECH Manufacturing Initiative (USA); B. J. Rice, J. Byers, SEMATECH (USA); Y. Avitan, R. Peltinov, M. Bar-zvi, O. Adan, Applied Materials (Israel); J. Swyers, Applied Materials (USA); R. Z. Shneck, Ben Gurion Univ. of the Negev (Israel) 
65182C Across-wafer CD uniformity control through lithography and etch process: experimental verification [6518-77]

Q. Zhang, Univ. of California, Berkeley (USA); C. Tang, Spansion, Inc. (USA); J. Cain, Advanced Micro Devices, Inc. (USA); A. Hui, T. Hsieh, N. Maccrae, Spansion Inc. (USA); B. Singh, Advanced Micro Devices, Inc. (USA); K. Poolla, C. J. Spanos, Univ. of California, Berkeley (USA)

65182D Image analysis of alignment and overlay marks with compound structure [6518-80] R. Chalykh, I. Pundaleva, J.-H. Shin, S.-S. Kim, H.-K. Cho, J.-T. Moon, Samsung Electronics Co. (South Korea)

65182E Methodical approach to improve defect detection sensitivity on lithography process using DUV inspection system [6518-81]

C. Lee, S. Won, D. Seo, H. Kim, J. Kim, Hynix Semiconductor, Inc. (South Korea); J.-H. Yeo, I. Dolev, C.-H. Kwak, Applied Materials (Israel)

65182F Etch process monitoring by electron beam wafer inspection [6518-82]

L. Lin, J.-Y. Chen, W.-Y. Wong, Powerchip Semiconductor Co. (Taiwan); M. McCord,

A. Tsai, S. Oestreich, I. De, J. Lauber, A. Kang, KLA-Tencor (USA)

65182G Immersion-induced defect SEM-based library for fast baseline improvement and excursion [6518-83]

I. Englard, Applied Materials Europe (Netherlands); R. Stegen, E. Van Brederode,

P. Vanoppen, I. Minnaert-Janssen, F. Duray, T. der Kinderen, G. Tanriseven, I. Lamers, M. B. Mantecon, ASML Netherlands B.V. (Netherlands); L. Levin, E. Binyamini, N. Raccah, S. Dror, E. Valfer, O. Rotlevi, Applied Materials Europe (Israel); R. Schreutelkamp, R. Piech, Applied Materials Europe (France)

$65182 \mathrm{H}$ Novel technology of automatic macro inspection for 32-nm node and best focus detection [6518-85]

K. Fukazawa, K. Endo, Nikon Corp. (Japan); K. Yoshino, Y. Yamazaki, Toshiba Corp. (Japan)

651821 Results from a new die-to-database reticle inspection platform [6518-86]

W. Broadbent, Y. Xiong, M. Giusti, R. Walsh, A. Dayal, KLA-Tencor Corp. (USA)

65182K Inspection sensitivity improvement through optimization of lobe blocking on high-end memory devices [6518-88]

C. Lee, S. Won, D. Seo, H. Kim, J. Kim, Hynix Semiconductor, Inc. (South Korea); J.-H. Yeo,

I. Dolev, C.-H. Kwak, Applied Materials (Israel)

65182L Use of automated EBR metrology inspection to optimize the edge bead process [6518-89]

A. Carlson, T. Le, A. Pai, Rudolph Technologies (USA); J. Hallen, B. Rioux, National

Semiconductor (USA)

$65182 \mathrm{M}$ High-throughput polarization imaging for defocus and dose inspection for production wafers [6518-90]

G. Sun, E. Onoichenco, Y. Fu, Y. Liu, R. Amell, C. McCandless, R. Reddy, G. Kumar,

M. Guest, Rudolph Technologies, Inc. (USA)

65182N Real-time spatial control of photoresist development rate [6518-91]

A. Tay, W.-K. Ho, N. Hu, C.-M. Kiew, National Univ. of Singapore (Singapore); K.-Y. Tsai,

National Taiwan Univ. (Taiwan) 
651820 A predictive method to forecast spatial variability of stochastic processes for deep nanoscale semiconductor manufacturing [6518-92]

Y. Chen, VIGMA Nanoelectronics (USA)

65182P Advanced process control for hyper-NA lithography based on CD-SEM measurement [6518-93]

T. Ishimoto, Hitachi High-Technologies Corp. (Japan) and IMEC (Belgium); K. Sekiguchi, Hitachi High Technologies Europe GmbH (Germany) and IMEC (Belgium); N. Hasegawa, T. Maeda, K. Watanabe, Hitachi High-Technologies Corp. (Japan); G. Storms, D. Laidler, S. Cheng, IMEC (Belgium)

65182Q Application of integrated scatterometry (iODP) to detect and quantify resist profile changes due to resist batch changes in a production environment [6518-94]

S. Ali, L. Chen, J. Tiffany, A. Yadav, Qimonda AG (USA); B. Swain, D. Dixon, S. Lickteig, Tokyo Electron America, Inc. (USA)

65182R Litho cell control using MPX [6518-95]

E. Apelgren, H. Kennemer, Spansion LLC (USA); C. Nelson, B. Eichelberger, J. Robinson, KLA-Tencor (USA)

65182S Data sharing system for lithography APC [6518-96]

E. Kawamura, Y. Teranishi, M. Shimabara, Fujitsu Ltd. (Japan)

65182T CD measurement in flash memory using substrate current technology [6518-97]

Y.-U, Ko, Fab Solutions, Inc. (USA); K. Yamada, T. Ushiki, Fab Solutions, Inc. (Japan)

$65182 \mathrm{U}$ Overlay metrology for dark hard mask process: simulation and experiment study [6518-99] J. Shin, R. Chalykh, H. Kang, S. Kim, S. Lee, H.-K. Cho, Samsung Semiconductor Research and Development (South Korea)

$65182 \mathrm{~V}$ In-chip overlay metrology of $45 \mathrm{~nm}$ and $55 \mathrm{~nm}$ processes [6518-100]

Y. S. Ku, C. H. Tung, Y. P. Li, H. L. Pang, Industrial Technology Research Institute (Taiwan);

C. M. Ke, Y. H. Wang, D. C. Huang, Taiwan Semiconductor Manufacturing Co. (Taiwan);

N. P. Smith, Nanometrics, Inc. (Taiwan); L. Binns, Nanometrics, Inc. (United Kingdom)

65182W Evaluation of AIM overlay mark for thin film head application [6518-101]

Y. Li, Seagate Technology (USA); A. Fan, KLA-Tencor Corp. (USA); G. Etheridge, G. Finken,

D. Louder, Seagate Technology (USA)

65182X Through-focus technique for overlay metrology [6518-102]

A.-S. Liu, Y.-S. Ku, Industrial Technology Research Institute (Taiwan); N. Smith, Nanometrics, Inc. (Taiwan)

65182Y Improvement of front-end process overlay in 60nm DRAM [6518-103]

Y.-S. Hwang, W.-K. Ma, E. Kang, C.-M. Lim, S.-C. Moon, Hynix Semiconductor Industries Co., Ltd. South Korea); S.-J. An, K. Rhe, ASML (South Korea)

$65182 Z$ Hardware, materials, and parameters optimization for improvement of immersion overlay [6518-104]

W.-K. Ma, Y.-S. Hwang, E.-K. Kang, S. Park, J.-H. Kang, C.-M. Lim, S.-C. Moon, Hynix

Semiconductor Industries Co., Ltd. (South Korea) 
$65183145 \mathrm{~nm}$ design rule in-die overlay metrology on immersion lithography processes [6518-106] Y.-H. Shih, G. K. C. Huang, C.-C. Yu, United Microelectronics Corp. (Taiwan); M. Adel, KLA-Tencor Israel (Israel); C.-C. K. Huang, KLA-Tencor Corp. (USA); P. Izikson, E. Kassel, KLA-Tencor Israel (Israel); S. Mathur, C.-J. Huang, D. Tien, KLA-Tencor Corp. (USA); Y. Avrahamov, KLA-Tencor Israel (Israel)

651832 Say good-bye to DOF: statistical process window analysis with inline lithographic process variations [6518-107]

W. Zhou, M. Tang, H. Koh, M. Zhou, Chartered Semiconductor Manufacturing, Ltd.

(Singapore)

651833 Use of in-line AFM as LWR verification tool in $45 \mathrm{~nm}$ process development [6518-108] M. H. Hsieh, K. H. Shi, J. H. Yeh, R. H. Hsu, M. Tsai, S. F. Tzou, United Microelectronics Corp. (Taiwan)

651834 Die-to-database verification tool for detecting CD errors, which are caused by OPC features, by using mass gate measurement and layout information [6518-109]

T. Kitamura, T. Hasebe, K. Kubota, F. Sakai, S. Nakazawa, D. Lin, M. J. Hoffman,

M. Yamamoto, NanoGeometry Research, Inc. (Japan); M. Inove, Topcon Corp. (Japan)

651835 Major trends in extending CD-SEM utility [6518-112]

B. Bunday, J. Allgair, International SEMATECH Manufacturing Initiative (USA); K. Yang,

S. Koshihara, H. Morokuma, Hitachi High-Technologies Corp. (Japan); A. Danilevsky,

C. Parker, L. Page, Hitachi High Technologies America, Inc. (USA)

651836 Stochastic simulation of material and process effects on the patterning of complex layouts [6518-115]

N. Tsikrikas, D. Drygiannakis, G. P. Patsis, G. Kokkoris, I. Raptis, E. Gogolides, Institute of Microelectronics (Greece)

651837 MacroCD contact ellipticity measurements for lithography tool qualification [6518-116]

I. Englard, Applied Materials Europe (Netherlands); E. van Setten, G.-J. Janssen,

P. Vanoppen, I. Minnaert-Janssen, F. Duray, ASML Netherlands B.V. (Netherlands); O. Adan, A. Moran, L. Gershtein, R. Peltinov, Applied Materials Europe (Israel)

651838 Sub-nanometer CD-SEM matching [6518-117]

T. Lott, R. J. Elias, Cypress Semiconductor (USA)

651839 Visible light angular scatterometry for nanolithography [6518-118]

R. M. Al-Assaad, L. Tao, W. Hu, The Univ. of Texas at Dallas (USA)

65183A Robust sub-50-nm CD control by a fast-goniometric scatterometry technique [6518-119] J. Hazart, P. Barritault, S. Garcia, CEA-LETI Minatec (France); T. Leroux, P. Boher, ELDIM (France); K. Tsujino, OMRON Corp. (Japan)

\section{Part Three}

65183B Accurate and reliable optical CD of MuGFET down to 10nm [6518-120]

P. Leray, G. F. Lorusso, S. Cheng, N. Collaert, M. Jurczak, IMEC (Belgium); S. Shirke, VLSI

Standard (USA) 
65183C OCD metrology by floating $\mathbf{n} / \mathbf{k}$ [6518-121]

S.-S. Yu, J. Huang, C.-M. Ke, T.-S. Gau, B. J. Lin, A. Yen, Taiwan Semiconductor Manufacturing Co., Ltd. (Taiwan); L. Lane, V. Vuong, Y. Chen, Timbre Technologies, Inc. (USA)

65183D High-resolution in-die metrology using beam profile reflectometry and ellipsometry [6518-123]

C. Jun, J. Park, Samsung Electronics Co., Ltd. (South Korea); J. Opsal, H. Pois, I.-K. Kim, J.-W. Kim, L. Nicolaides, Therma-Wave, Inc. (USA)

65183E The study to enhance the mask global CD uniformity by removing local CD variation [6518-126]

Y. Choi, M. Kim, O. Han, Hynix Semiconductor (South Korea)

65183F Achievement of sub nanometer reproducibility in line scale measurements with the nanometer comparator [6518-128]

R. Köning, J. Flügge, H. Bosse, Physikalisch-Technische Bundesanstalt (Germany)

65183G Aera193i: aerial imaging mask inspection for immersion lithography [6518-129]

Y. Zabar, C. Braude, S. Mangan, Applied Materials (Israel); D. Rost, R. Mann, MP Mask Technology Ctr., LLC (USA)

$65183 \mathrm{H}$ Critical dimension measurements on phase-shift masks using an optical pattern placement metrology tool [6518-130]

H. Bittner, D. Adam, J. Bender, A. Boesser, M. Heiden, K. Roeth, Vistec Semiconductor Systems GmbH (Germany)

65183J Novel CD-SEM calibration reference consisting of 100-nm pitch grating and positional identification mark [6518-133]

Y. Nakayama, Hitachi, Ltd. (Japan); H. Kawada, S. Yoneda, Hitachi High-Technologies Corp. (Japan); T. Mizuno, Hitachi High-Tech Science Systems Corp. (Japan)

65183K Advanced CD-AFM probe tip shape characterization for metrology accuracy and throughput [6518-134]

H.-C. Liu, J. R. Osborne, M. Osborn, G. A. Dahlen, Veeco Instruments, Inc. (USA)

65183L An advanced AFM sensor: its profile accuracy and low probe wear property for high aspect ratio patterns [6518-137]

M. Watanabe, S. Baba, T. Nakata, Hitachi, Ltd. (Japan); T. Kurenuma, Y. Kunitomo, M. Edamura, Hitachi Kenki FineTech Co., Ltd. (Japan)

$65183 \mathrm{M} \quad$ New inline AFM metrology tool suited for LSI manufacturing at the 45-nm node and beyond [6518-139]

M. Edamura, Y. Kunitomo, T. Morimoto, S. Sekino, T. Kurenuma, Y. Kembo, Hitachi Kenki FineTech Co., Ltd. (Japan); M. Watanabe, S. Baba, K. Hidaka, Hitachi, Ltd. (Japan)

65183N Magnification calibration standards for sub-100nm metrology [6518-140] S. Deo, Univ. of Tennessee (USA); D. Joy, Univ. of Tennessee (USA) and Oak Ridge National Lab. (USA)

651830 Advances in CD-AFM scan algorithm technology enable improved CD metrology [6518-141]

L. Mininni, Veeco Instruments (USA); J. Foucher, P. Faurie, CEA/LETI/MINATEC (France) 
65183P Plasma-assisted cleaning by electrostatics (PACE) [6518-142]

W. M. Lytle, H. Shin, D. N. Ruzic, Univ. of Illinois at Urbana-Champaign (USA)

65183Q Optical characterization of microstructures of high aspect ratio [6518-143]

T. Tamulevicius, S. Tamulevicius, M. Andrulevicius, G. Janusas, V. Ostasevicius, A. Palevicius, Kaunas Univ. of Technology (Lithuania)

65183R Contrarian approach to and ultimate solution for 193nm reticle haze [6518-144]

O. Kishkovich, A. Grayfer, F. V. Belanger, Entegris, Inc. (USA)

651835 Thermal imaging of a lithography cell, including exposure tool, using a self-contained instrumented wafer [6518-145]

Z. Reid, M. Wiltse, S. Burgan, G. Roche, SensArray Corp. (USA)

65183T Optimization of lithography process to improve image deformation of contact hole sub-90 nm technology node [6518-146]

S. Jun, J. Kim, E. Jeong, Y. Yun, J. Kim, K. Kim, DongbuElectronics (South Korea)

65183V Resolution enhancement technique using oxidation process with nitride hardmask process [6518-148]

E. Jeong, J. Kim, K. Kim, D. Kim, H. Lim, DongbuElectronics (South Korea)

65183W Enhanced hole shape of flash devices in ArF lithography by eliptical mask bias technique [6518-150]

Y.-D. Jeon, S. Jun, J.-H. Kang, S.-U. Lee, J. Kim, K. Kim, DongbuElectronics (South Korea)

65183 Purge micro-environment with ionized air to reduce chances of ESD damages to wafers [6518-153]

H. Wang, Y. Liu, Entegris, Inc. (USA); M. Cisewski, Consultant (USA)

651832 Monitoring airborne molecular contamination: a quantitative and qualitative comparison of real-time and grab-sampling techniques [6518-154]

A. M. Shupp, D. Rodier, S. Rowley, Particle Measuring Systems, Inc. (USA)

651841 Optimizing surface finishing processes through the use of novel solvents and systems [6518-156]

M. Quillen, P. Holbrook, Eastman Chemical Co. (USA); J. Moore, DAETEC, LLC (USA)

651842 New filter media development for effective control of trimethysilanol (TMS) and related low molecular weight silicon containing organic species in the photobay ambient [6518-157]

A. Grayfer, F. V. Belanger, P. Cate, D. Ruede, Entegris Inc. (USA)

651843 Non-contacting electrostatic voltmeter for wafer potential monitoring [6518-158]

M. A. Noras, W. A. Maryniak, Trek, Inc. (USA)

651844 Optimized molecular contamination monitoring for lithography [6518-159]

D. Rodier, Particle Measuring Systems (USA)

651845 The novel advanced process control to eliminate AICu-PVD induced overlay shift [6518-160]

C. H. Huang, C. C. Yang, E. Yang, T. H. Yang, K. Chen, J. Ku, C. Y. Lu, Macronix International

Co., Ltd. (Taiwan) 
651846 Low-pressure drop filtration of airborne molecular organic contaminants using openchannel networks [6518-161]

A. J. Dallas, J. Joriman, L. Ding, G. Weineck, K. Seguin, Donaldson Co., Inc. (USA)

651847 Novel method of under-etch defect detection for contact layers based on Si substrate using optic wafer inspection tools [6518-162]

B.-H. Lee, J.-S. Choi, S.-B. Chin, D.-H. Cho, C.-L. Song, Samsung Electronics Co., Ltd. (South Korea)

651848 CD-bias evaluation and reduction in CD-SEM linewidth measurements [6518-163] M. Tanaka, C. Shishido, W. Nagatomo, Hitachi, Ltd. (Japan); K. Watanabe, Hitachi HighTechnologies Corp. (Japan)

651849 Ellipsometric studies of the absorption of liquid by photo resist [6518-164] H. Jeong, J. Kyung, S. Park, K. Lee, H. Lee, H. Cheon, I. An, Hanyang Univ. (South Korea); S. Lee, Samsung Eletronics Co., Ltd. (South Korea)

65184A Advanced defect definition method using design data [6518-165]

K. Lim, D. Patel, International SEMATECH Manufacturing Initiative (USA); K. Yang, S. Koshihara, Hitachi High-Technologies Corp. (Japan); L. Page, A. Self, M. Martinez, Hitachi High-Technologies America, Inc. (USA)

65184B Image quality improvement in inspection systems using double integrator illumination [6518-166]

A. Takada, H. Suzuki, T. Tojo, Topcon Corp. (Japan); M. Shibuya, Tokyo Polyłechnic Univ. (Japan)

65184C Study of ADI (After Develop Inspection) on photo resist wafers using electron beam (II) [6518-167]

T. Hayashi, M. Saito, K. Fujihara, S. Shibuya, Y. Kudou, Tokyo Electron, Ltd. (Japan);

H. Nagaike, Tokyo Electron AT, Ltd. (Japan); J. Lin, J. Jau, Hermes Microvision, Inc. (USA)

65184D 3D anisotropic semiconductor grooves measurement simulations (scatterometry) using FDTD methods [6518-168]

H. Shirasaki, Tamagawa Univ. (Japan)

65184E Metrology of replicated diffractive optics with Mueller polarimetry in conical diffraction [6518-169]

T. Novikova, A. De Martino, P. Bulkin, Q. Nguyen, B. Drévillon, LPICM, École Polytechnique (France); V. Popov, A. Chumakov, Moscow State Univ. (Russia)

65184F Influence of wafer warpage on photoresist film thickness and extinction coefficient measurements [6518-170]

X. WU, A. Tay, National Univ. of Singapore (Singapore)

65184G Angular scatterometry for line-width roughness measurement [6518-171] D.-M. Shyu, Y.-S. Ku, Industrial Technology Research Institute (Taiwan); N. Smith, Nanometrics, Inc. (Taiwan)

$65184 \mathrm{H}$ Charging measurement using SEM embedded energy filter [6518-172] F. Levitov, A. Karabekov, G. Eytan, Applied Materials Israel (Israel); G. Golan, Holon Institute of Technology (Israel) and The Open Univ. (Israel) 
651841 Contact leakage and open monitoring with an advanced e-beam inspection system [6518-173]

S. Lei, H. Liu, M. Tsai, United Microelectronics Corp. (Taiwan); H.-C. Wu, Hermes Microvision, Inc. (Taiwan); H. Xiao, J. Jau, Hermes Microvision, Inc. USA (USA)

65184J An approach to modeling and on-line identification for piezoelectric stack actuator [6518-174]

Y. Wang, X. Zhao, W. Chu, Harbin Institute of Technology (China)

65184K The optimization of photoresist profile for sub-90nm technology [6518-175]

H. Jeon, C. Shim, J. Hong, J. Han, K. Kim, Dongbu Electronics (South Korea)

65184L Study on micro-bubble defect induced by RRC coating [6518-176]

Y.-H. Liu, W.-S. Liao, H.-H. Lin, C.-J. Chen, C. C. Huang, United Microelectronics Corp. (Taiwan)

$65184 \mathrm{M}$ Characterization of resist thinning and profile changes using scatterometry [6518-178] J. Fullam, K. Petrillo, IBM Corp. (USA)

65184N Contamination removal from collector optics and masks: an essential step for nextgeneration lithography [6518-179]

H. Shin, S. N. Srivastava, D. N. Ruzic, Univ. of Illinois at Urbana-Champaign (USA)

65184P A new SEM CD operator verified against Monte Carlo simulations [6518-181]

C. G. Frase, D. Gnieser, Physikalisch-Technische Bundesanstalt (Germany); K. Dirscherl, Danish Fundamental Metrology, Ltd. (Denmark); E. Buhr, H. Bosse, Physikalisch-Technische Bundesanstalt (Germany)

65184Q Characterization and adjustment of high performance objectives for DUV applications [6518-182]

S. Müller-Pfeiffer, L. Körner, S. Franz, O. R. Falkenstörfer, H. Lauth, JENOPTIK Laser, Optik, Systeme GmbH (Germany)

65184R Phame: a novel phase metrology tool of Carl Zeiss for in-die phase measurements under scanner relevant optical settings [6518-183]

S. Perlitz, U. Buttgereit, T. Scherübl, Carl Zeiss SMS GmbH (Germany)

651845 Non-linear methods for overlay control [6518-184]

M. Kupers, D. Choi, B. Habets, Qimonda Dresden GmbH and Co. OHG (Germany);

G. Simons, E. Wallerbos, ASML Netherlands B.V. (Netherlands)

65184T Leveraging LER to minimize linewidth measurement uncertainty in a calibration exercise [6518-185]

J. Robert, B. Banke, IBM Microelectronics (USA); R. Dixson, National Institute of Standards and Technology (USA)

$65184 \mathrm{~V}$ Köhler illumination analysis for high-resolution optical metrology using $193 \mathrm{~nm}$ light [6518-187]

Y.-J. Sohn, R. M. Silver, National Institute of Standards and Technology (USA)

65184W Critical dimension: MEMS road map [6518-188]

M. Poulingue, Nanometrics France (France); P. Knutrud, Nanometrics (USA) 
65184X Enabling gate etch process development using scatterometry [6518-189]

J. Koshy, M. Sendelbach, IBM Microelectronics (USA); P. Herrera, KLA-Tencor Corp. (USA)

$65184 Z$ ArF pellicle degradation mechanism for resolving CD variation [6518-191]

H. Choi, Y. Ahn, J. Ryu, Y. Lee, B. An, S. Lee, Samsung Electronics Co., Ltd. (South Korea)

651850 Effect and procedures of post exposure bake temperature optimization on the CD uniformity in a mass production environment [6518-192]

K. Ruck, H. Weichert, Tokyo Electron Europe, Ltd. (Germany); S. Hornig, F. Finger,

G. Fleischer, Qimonda Dresden GmbH and Co. OHG (Germany); D. Hetzer, Timbre Technologies, Inc. (USA)

651851 Scatterometry characterization of polysilicon gate profiles in a $90 \mathrm{~nm}$ logic process [6518-193]

E. B. Maiken, Intel Corp. (USA)

651855 Scatterometry solutions and vision for advanced lithography process control [6518-199]

T. Levin, M. Livne, Intel Corp. (Israel); R. M. Gillespie, Intel Corp. (USA)

651856 Scatterometry measurement of nested lines, dual space, and rectangular contact CD on phase-shift masks [6518-200]

K. M. Lee, Intel Corp. (USA); S. Yedur, Timbre Technologies, Inc. (USA); S. Henrichs,

M. Tavassoli, K. Baik, Intel Corp. (USA)

651857 Implementation strategies and return on investment for integrated CD control [6518-201]

L. Lane, B. Monteverde, Timbre Technologies, Inc. (USA)

Author Index

xviii 


\title{
Symposium Committees
}

\author{
Symposium Chair
}

Roxann L. Engelstad, University of Wisconsin/Madison (USA)

Symposium Cochair

Christopher J. Progler, Photronics, Inc. (USA)

Technical Organizing Committees

Executive Committee

John A. Allgair, SEMATECH, Inc. (USA) and Freescale Semiconductors (USA)

Chas N. Archie, IBM Corporation (USA)

Roxann L. Engelstad, University of Wisconsin/Madison (USA)

Donis G. Flagello, ASML US, Inc. (USA)

Clifford L. Henderson, Georgia Institute of Technology (USA)

Michael J. Lercel, SEMATECH (USA) and IBM Corporation (USA)

Harry J. Levinson, Advanced Micro Devices, Inc. (USA)

Qinghuang Lin, IBM Thomas J. Watson Research Center (USA)

Christopher J. Progler, Photronics, Inc. (USA)

Franklin M. Schellenberg, Mentor Graphics Corporation (USA)

Vivek K. Singh, Intel Corporation (USA)

Alfred K. K. Wong, Magna Design Automation, Inc. (USA)

Advisory Committee

Robert D. Allen, IBM Almaden Research Center (USA)

William H. Arnold, ASML Netherlands B.V. (Netherlands)

Timothy A. Brunner, IBM Thomas J. Watson Research Center (USA)

Ralph R. Dammel, AZ Electronic Materials USA Corporation (USA)

Roderick R. Kunz, MIT Lincoln Laboratory (USA)

Harry J. Levinson, Advanced Micro Devices, Inc. (USA)

Burn Lin, Taiwan Semiconductor Manufacturing Company, Ltd. (Taiwan)

Chris A. Mack, LithoGuru.com

Victor Pol, Freescale Semiconductor, Inc. (USA)

Michael T. Postek, National Institute of Standards and Technology (USA)

Luc Van den Hove, IMEC (Belgium)

C. Grant Willson, The University of Texas at Austin (USA)

Anthony Yen, Taiwan Semiconductor Manufacturing Company, Ltd.

(Taiwan) and Cymer, Inc. (USA) 
Downloaded From: https://www.spiedigitallibrary.org/conference-proceedings-of-spie on 26 Apr 2023

Terms of Use: https://www.spiedigitallibrary.org/terms-of-use 


\title{
Conference Committee
}

\author{
Conference Chair
}

Chas N. Archie, IBM Microelectronics (USA)

Conference Cochair

John A. Allgair, SEMATECH, Inc. (USA)

Program Committee

Ofer Adan, Applied Materials (Israel)

Michael J. Anderson, Rohm and Haas Electronic Materials (USA)

Jason P. Cain, Advanced Micro Devices, Inc. (USA)

Alain G. Deleporte, STMicroelectronics (France)

Daniel J. C. Herr, Semiconductor Research Corporation (USA)

David C. Joy, The University of Tennessee (USA)

Chih-Ming Ke, Taiwan Semiconductor Manufacturing Co., Ltd. (Taiwan)

Byoung-Ho Lee, Samsung Electronics Co., Ltd. (South Korea)

Christopher J. Raymond, Nanometrics, Inc. (USA)

Martha I. Sanchez, IBM Almaden Research Center (USA)

Richard M. Silver, National Institute of Standards and Technology (USA)

Bhanwar Singh, Advanced Micro Devices, Inc. (USA)

Michael W. Stan, MicroChem Corporation (USA)

Alexander Starikov, Intel Corporation (USA)

Neal T. Sullivan, Soluris, Inc. (USA)

Brian M. Trafas, KLA-Tencor Corporation (USA)

Vladimir A. Ukraintsev, Texas Instruments, Inc. (USA)

\section{Session Chairs}

1 Keynote Session

Chas N. Archie, IBM Microelectronics (USA)

2 Process Control I: Lithography Control

Christopher J. Raymond, Nanometrics, Inc. (USA)

3 CD for Development I: OPC and Metrology

Neal T. Sullivan, Soluris, Inc. (USA)

Chih-Ming Ke, Taiwan Semiconductor Manufacturing Co., Ltd. (Taiwan)

Chas N. Archie, IBM Microelectronics (USA)

4 Overlay

Alexander Starikov, Intel Corporation (USA)

Daniel J. C. Herr, Semiconductor Research Corporation (USA) 
5 CDSEM: Techniques, Limits, Etc.

David C. Joy, The University of Tennessee (USA)

Byoung-Ho Lee, Samsung Electronics Company, Ltd. (South Korea)

6 Inspection

Brian M. Trafas, KLA-Tencor Corporation (USA)

Michael W. Stan, MicroChem Corporation (USA)

$7 \quad$ Scatterometry Techniques, Limits, Etc.

Christopher J. Raymond, Nanometrics, Inc. (USA)

Jason P. Cain, Advanced Micro Devices, Inc. (USA)

8 Standards and Techniques

Bhanwar Singh, Advanced Micro Devices, Inc. (USA)

Vladimir A. Ukraintsev, Texas Instruments, Inc. (USA)

9 Reference Metrology and Instruments

Richard M. Silver, National Institute of Standards and Technology (USA)

Michael J. Anderson, Rohm and Haas Electronic Materials (USA)

10 Metrology Challenges

Alexander Starikov, Intel Corporation (USA)

Ofer Adan, Applied Materials (Israel)

11 New Trends in Metrology

David C. Joy, The University of Tennessee (USA)

Vladimir A. Ukraintsev, Texas Instruments, Inc. (USA)

12 Line-edge and Line-width Roughness

Bhanwar Singh, Advanced Micro Devices, Inc. (USA)

Neal T. Sullivan, Soluris, Inc. (USA)

13 Mask Metrology

Martha I. Sanchez, IBM Almaden Research Center. (USA)

Daniel J. C. Herr, Semiconductor Research Corporation (USA)

14 Process Control II

Brian M. Trafas, KLA-Tencor Corporation (USA)

Jason P. Cain, Advanced Micro Devices, Inc. (USA)

15 CD for Development II

John A. Allgair, SEMATECH, Inc. (USA)

Chas N. Archie, IBM Microelectronics (USA)

Poster Session

Christopher J. Raymond, Nanometrics, Inc. (USA) 